\title{
Anesthetic Management of a Case of Coarctation of Aorta Taken Up for Intracranial Aneurysm Coiling
}

\author{
Shamik Paul ${ }^{1}$ Summit D. Bloria ${ }^{1} \quad$ Hemant Bhagat ${ }^{1} \quad$ Ankur Luthra $^{1}$ \\ ${ }^{1}$ Department of Anesthesia, Postgraduate Institute of Medical \\ Education and Research, Chandigarh, India \\ Address for correspondence Summit D. Bloria, MD, DNB, Department \\ of Anesthesia, Postgraduate Institute of Medical Education and \\ Research, Chandigarh, India (e-mail: summitbloria13@gmail.com).
}

J Neuroanaesthesiol Crit Care 2020;7:38-40

\begin{abstract}
We describe our experience of management of a young hypertensive male taken up for coiling of an unruptured intracranial aneurysm whom we diagnosed to be a Keywords

- intracranial aneurysm

- coarctation of aorta

- aneurysm coiling case of coarctation of aorta during preanesthetic check-up. This diagnosis changed the treatment of the patient completely. We report this to emphasize the need to do a thorough preoperative check-up in every case. We also touch upon the important anesthetic considerations to be observed while managing such cases.
\end{abstract}

\section{Introduction}

The estimated incidence of intracranial aneurysms (IA) is generally $2 \%$ of the general western population. ${ }^{1,2}$ Coarctation of aorta $(\mathrm{COA})$ is an evident major risk factor for IA formation. CoA accounts for $5 \%$ of all cardiac defects. ${ }^{3}$ The coincidence of IAs with CoA ranges from $2 \%$ to as high as $50 \%{ }^{4}$ The estimated incidence of COA in patients with IAs is low $(0.19-1.9 \%) .{ }^{5,6}$ We present our experience of successful anesthetic management of a 26-year-old male diagnosed with $\mathrm{CoA}$ and posted for endovascular coiling of an unruptured internal carotid artery (ICA) aneurysm.

\section{Case Report}

A young male, smoker, and known hypertensive for last 3 months on three antihypertensives (prescribed by a local pharmacist) presented to this hospital with sudden onset weakness of right upper and lower limbs, headache, and multiple episodes of vomiting. His computed tomography (CT) scan head was performed on emergent basis which showed left basal ganglia bleed. It was decided to manage the basal ganglia bleed conservatively. His CT angiography revealed an unruptured partially thrombosed saccular aneurysm measuring $5.9 \times 4.5 \mathrm{~mm}$ with neck measuring $4.9 \mathrm{~mm}$ arising from the dorsal wall of left supra clinoid ICA. As the location of the aneurysm had a difficult surgical access, the patient was subsequently advised coiling of the unruptured aneurysm.

The patient was referred to us for preanesthetic check-up (PAC) where the patient was restless, having severe headache. His muscle power was $5 / 5$ on right side and $0 / 5$ on left side. His blood pressure (BP) was 220/130 mm Hg and patient was being prescribed amlodipine $10 \mathrm{mg}$ (tablet), telmisartan (40 mg, tablet), hydrochlorothiazide $(12.5 \mathrm{mg}$ ) and metoprolol (tablet) extended release $20 \mathrm{mg}$ once daily along with Labetalol injection on SOS basis. We found that his lower pulses were feeble, and when we examined his BP in lower limb, it was $112 / 80 \mathrm{~mm} \mathrm{Hg}$. On auscultation of the heart, there was a systolic murmur heard on the precordium. Electrocardiography was suggestive of left ventricular hypertrophy (LVH) (- Fig. 1). Upon suspicion of CoA, it was advised that the patient undergoes a chest $\mathrm{CT}$ angiogram and cardiac workup. Diagnostic digital subtraction angiography (DSA) confirmed the CoA distal to the origin of left subclavian artery (-Fig. 2) with very prominent collaterals. The morphology of ICA aneurysm was also confirmed and the coiling was deferred till cardiac evaluation of the patient.

The patient was subsequently examined by cardiology team and echocardiogram (echo) test revealed concentric LVH with normal left ventricular systolic function and an ejection fraction of $82 \%$. Cardiologist opined that the ICA aneurysm be coiled before correction of CoA keeping in view the hemodynamic fluctuations that may occur during correction of coarctation (predisposing to aneurysm rupture). We subsequently reviewed the patient for coiling under general anesthesia with high cardiac risk.

On the day of surgery, patient was shifted to DSA suite and baseline monitors were attached. The patient was drowsy preoperatively. His left radial artery was cannulated under local anesthesia and invasive BP transduced, which was $187 / 97 \mathrm{~mm} \mathrm{Hg}$. Noninvasive BP cuff was attached in left calf and cycled at $1 \mathrm{~min}$ and was found to be $118 / 78 \mathrm{~mm} \mathrm{Hg}$. 


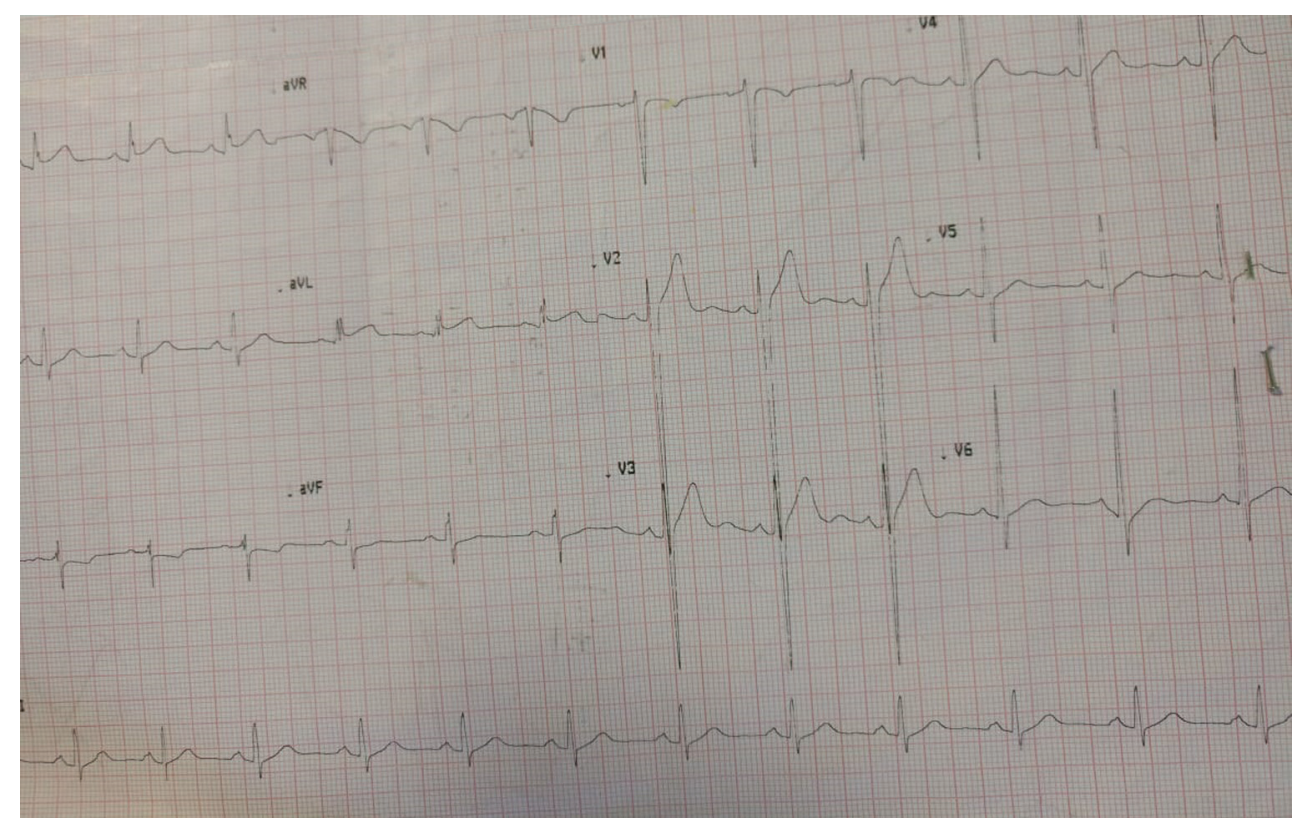

Fig.1 Electrocardiogram suggestive of left ventricular hypertrophy.

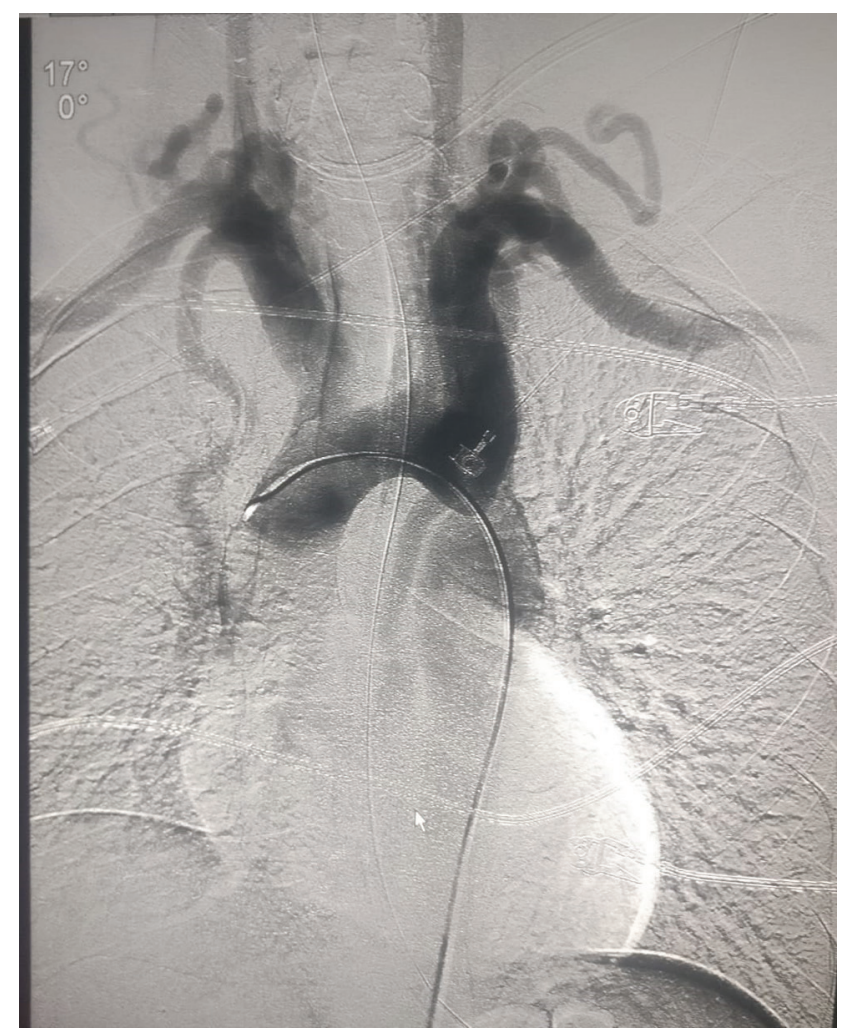

Fig. 2 Coarctation of aorta as seen during digital subtraction angiography.

Our intraoperative aim was to prevent wide fluctuations in the hemodynamics and prevent hyperemia of the cerebral circulation and regions above the level of coarctation and also in the same time prevent ischemia below the coarctation. The patient was induced with morphine $15 \mathrm{mg}$ injection, fentanyl $100 \mu \mathrm{g}$ injection, and thiopentone $175 \mathrm{mg}$ injection. After ensuring adequate mask ventilation, vecuronium $6 \mathrm{mg}$ injection intravenously was given followed by lignocaine injection (preservative free) $60 \mathrm{mg}$ and esmolol $30 \mathrm{mg}$ and patient intubated with size $8.5-\mathrm{mm}$ internal diameter (ID) endotracheal tube. Thereafter, balanced anesthesia was administered to the patient with oxygen and nitrous oxide in the ratio $1: 1$ and sevoflurane to attain a minimum alveolar concentration (MAC) of 0.8 to 1.0. We then placed an arterial cannula in left dorsalis pedis artery and transduced it to measure BP distal to the coarctation. A central venous catheter was placed aseptically in right subclavian. Thereafter, the patient was handed over to the neuroradiologist for endovascular coiling.

The intervention proceeded uneventfully and patient's BP in lower limb was kept above a mean arterial pressure (MAP) above $70 \mathrm{~mm} \mathrm{Hg}$ throughout the procedure. The MAP in the left radial artery remained around $110 \mathrm{~mm}$ $\mathrm{Hg}$ during the procedure. The urine output was monitored hourly. Postoperatively, patient was shifted to neurointensive care unit unreversed and electively ventilated. He was slowly weaned off the ventilator and extubated smoothly with a Glasgow Coma Scale of E4V5M6.

Two weeks post coiling of IA, the patient underwent correction of coarctation by balloon angioplasty uneventfully. He was discharged from the hospital with a residual weakness of $2 / 5$ on left side of body.

\section{Discussion}

The presence of the CoA (post ductal) is responsible for hyper tension and subsequent formation of intracerebral aneurysms. The cause of the basal ganglia bleed was in all probability a hypertensive bleed. As pressure in the precoarctation segment 
of the aorta increases, there is a significant elevation in the aortocerebral blood flow and coupled with the increased resistance of the cerebral circulation, the dilatation of the cervicocephalic arteries takes place. ${ }^{7}$ This scenario was very evident in our case.

In our patient, as the aortocranial pressure difference increased (due to coarctation) and with the presence of dilated cerebral as well as cervicocephalic arteries, the blood flow was increased proximally. In the distal segment however, the stenosis of the coarctation was responsible for decreased flow in the lower extremities leading to lower BP distal to the coarctation.

Our aim was to administer general anesthesia with aggressive hemodynamic monitoring to prevent any perioperative aneurysmal rupture and at the same time prevent any ischemia distal to the aneurysm. The obtundation of the sympathetic response was achieved by high opiate preinduction dose and administration of preservative free lignocaine and esmolol. Extubation of such patients also requires to be done in a controlled environment as any bucking can prove to be detrimental.

The DSA suite itself is a challenging place to administer anesthesia. The lack of space coupled with absence of expert assistance due to remoteness of the place gives us added challenges.

Our take-home messages while presenting this report is to stress on the following issues:

1. Any trivial finding during PAC must be thoroughly investigated, as the management of cases can completely change.
2. Understanding the altered physiology in patients of CoA needs to be understood while caring for these patients perioperatively.

\section{Conflict of Interest}

None declared.

\section{References}

1 Atkinson JL, Sundt TM Jr, Houser OW, Whisnant JP. Angiographic frequency of anterior circulation intracranial aneurysms. J Neurosurg 1989;70(4):551-555

2 Inagawa T. Trends in incidence and case fatality rates of aneurysmal subarachnoid hemorrhage in Izumo City, Japan, between 1980-1989 and 1990-1998. Stroke 2001;32(7): 1499-1507

3 Pádua LM, Garcia LC, Rubira CJ, de Oliveira Carvalho PE. Stent placement versus surgery for coarctation of the thoracic aorta. Cochrane Database Syst Rev 2012;5(5):CD008204

4 Shearer WT, Rutman JY, Weinberg WA, Goldring D. Coarctation of the aorta and cerebrovascular accident: a proposal for early corrective surgery. J Pediatr 1970;77(6):1004-1009

5 Fukuda H, Sako K, Yonemasu Y. Coarctation of the descending aorta with aneurysm of the anterior communicating artery. Surg Neurol 1985;23(4):380-382

6 Orsi P, Rosa G, Liberatori G, Lunardi PP, Ferrante L. Repair of two unruptured intracranial aneurysms in the presence of coarctation of the aorta-anesthetic implications and management. J Neurosurg Anesthesiol 1993;5(1):48-51

7 Rowe GG, Castillo CA, Afonso S, Young WP, Crumpton CW. Cerebral blood flow in coarctation of the aorta. J Clin Invest 1964;43:1922-1927 